\title{
Relationship between pore size and the gas pressure dependence of the gaseous thermal conductivity
}

\author{
G. Reichenauer*, U. Heinemann, H.-P. Ebert \\ Bavarian Center for Applied Energy Research, Am Hubland, 97074 Wuerzburg, Germany
}

Received 20 June 2006; received in revised form 12 January 2007; accepted 16 January 2007

Available online 20 January 2007

\begin{abstract}
In porous materials, such as foams or pressed powder boards, the thermal conductivity via the gas phase represents at ambient temperatures a significant or even dominant contribution to the total thermal conductivity. The variation of the thermal conductivity of an open porous material with gas pressure is a function of the pore size. In the pressure range below 1 bar, measurement of thermal conductivity offers a non-destructive probe of pore sizes larger than $100 \mathrm{~nm}$. If the thermal conductivity setup also allows for measurements at higher pressures, the pore size range can be extended to even smaller values.

Experimental data for a variety of different porous materials such as open porous foams, granular porous and nonporous materials and aerogels, porous solids with an adjustable mean pore size in the meso- or macro-pore range, are presented. The pore characteristics determined from thermal conductivity measurements are compared to morphological data derived from nitrogen sorption and small angle X-ray scattering data.

The potential and limits of this method in terms of the extraction of an average pore size and a pore size distribution are discussed.

(C) 2007 Elsevier B.V. All rights reserved.
\end{abstract}

Keywords: Thermal conductivity; Gas transport; Porous materials; Pore size

\section{Introduction}

The thermal conductivity as a function of gas pressure is a property that is closely related to the open porosity and the pore size of a porous solid. In particular, thermal transport via the gas phase significantly changes with gas pressure when the pore size is on the order of the mean free path of the gas molecules. Since the mean free path varies with gas pressure, a systematic pressure variation can be used to probe the pore size of an open porous system. For gas pressures below 1 bar, the gas transport is sensitive to pores of about $100 \mathrm{~nm}$ and above. The gas pressure dependence of the thermal conductivity can therefore be used to investigate porosity that is typically analysed by $\mathrm{Hg}$-porosimetry, a well established technique for the determination of pore size distributions in the micron to nanometer range. However, the latter method clearly fails when being applied to compliant materials that are compressed, rather

\footnotetext{
* Corresponding author. Tel.: +49 93170564 28; fax: +49 9317056460 .

E-mail address: Reichenauer@zae.uni-wuerzburg.de (G. Reichenauer).
}

than being infiltrated by the mercury upon analysis [1,2], thus yielding highly erroneous pore size distributions. Similar effects caused by the capillary pressure upon condensation are found upon nitrogen sorption analysis of compliant materials [3]. The measurement of the thermal conductivity as a function of gas pressure might therefore be an alternative, non-destructive technique to deduce the pore size of meso- and macro-porous materials.

With respect to applications of porous materials for thermal insulation, information on the gas pressure dependence of the thermal conductivity can be exploited to suppress the gaseous contribution to the heat transfer [4] and thus to provide high performance thermal insulation systems or to switch material from thermally conductive to insulating [5].

In this paper we present and discuss the application of analyzing the thermal conductivity of open porous materials as a function of gas pressure to extract information on the pore size. The results for different types of samples are compared to values derived via nitrogen sorption and small angle X-ray scattering. 


\section{Nomenclature}

a thermal diffusivity $\left(\mathrm{m}^{2} / \mathrm{s}\right)$

A cross sectional area of the sample perpendicular to the heat flow $\left(\mathrm{m}^{2}\right)$

$c_{p} \quad$ specific heat at constant pressure $(\mathrm{J} /(\mathrm{kg} \mathrm{K}))$

$d \quad$ pore size $(\mathrm{m})$

$d_{0} \quad$ thickness of sample (m)

$d_{\text {SAXS }}$ pore size determined via external surface area from SAXS data $(\mathrm{m})$

$D \quad$ average pore size determined from pressure dependence of the gaseous thermal conductivity (m)

$D^{\prime} \quad$ pore size variable in convolution (m)

$\mathrm{F}$ ratio of thermal conductivity for a material with a Gaussian distributed pore size and a system with only one pore size

Kn Knudsen number

$\ell \quad$ mean free path $(\mathrm{m})$

$\ell_{\text {gas }} \quad$ mean free path at reference pressure $p_{0}(\mathrm{~m})$

$N \quad$ normalization constant $\left(\mathrm{m}^{-1}\right)$

$p \quad$ gas pressure $(\mathrm{Pa})$

$p_{1 / 2} \quad$ gas pressure at half of the full gaseous thermal conductivity $(\mathrm{Pa})$

$p_{0} \quad$ reference gas pressure (here: $\left.1000 \mathrm{hPa}\right)(\mathrm{Pa})$

$P^{\prime} \quad$ electrical power for heating $(\mathrm{J} / \mathrm{s})$

$\Delta T \quad$ temperature difference across the sample $(\mathrm{K})$

$V_{\mathrm{p}} \quad$ specific meso- and macro-pore volume $\left(\mathrm{m}^{3} / \mathrm{kg}\right)$

$S_{\text {ext }} \quad$ specific external surface area $\left(\mathrm{m}^{2} / \mathrm{kg}\right)$

\section{Greek letters}

$\beta \quad$ constant

$\Phi \quad$ meso- and macro-porosity

$\lambda \quad$ thermal conductivity $(\mathrm{W} /(\mathrm{m} \mathrm{K}))$

$\lambda_{\text {total }}$ total thermal conductivity $(\mathrm{W} /(\mathrm{m} \mathrm{K}))$

$\lambda_{\text {solid }}$ thermal conductivity along the solid phase $(\mathrm{W} /(\mathrm{m} \mathrm{K}))$

$\lambda_{\text {radiative }}$ thermal conductivity via radiation $(\mathrm{W} /(\mathrm{m} \mathrm{K}))$

$\lambda_{\text {gas }}$ thermal conductivity via the gas phase $(\mathrm{W} /(\mathrm{m} \mathrm{K}))$

$\lambda_{\text {coupling thermal conductivity due to coupling effects }}$ $(\mathrm{W} /(\mathrm{m} \mathrm{K}))$

$\lambda_{\text {gas, } 0} \quad$ thermal conductivity of the free gas (not including convectional effects) $(\mathrm{W} /(\mathrm{m} \mathrm{K}))$

$\rho \quad$ density of the sample $\left(\mathrm{kg} / \mathrm{m}^{3}\right)$

$\rho_{\text {skeleton }}$ density of the solid phase within the sample $\left(\mathrm{kg} / \mathrm{m}^{3}\right)$

$\sigma \quad$ standard deviation of the Gaussian pore size distribution (m)

\section{Theoretical background}

\subsection{Gas pressure dependence of the gaseous thermal conductivity}

A good approximation to the thermal conductivity of a porous material can be written as the sum of four terms: $\lambda_{\text {total }}=\lambda_{\text {solid }}+\lambda_{\text {radiative }}+\lambda_{\text {gas }}+\lambda_{\text {coupling }}$,

where $\lambda_{\text {total, solid, radiative, gas }}$ are, respectively, the overall thermal conductivity, the heat transport via the solid phase, the transport via radiation, and the contribution due to the heat transfer via the gas phase; $\lambda_{\text {coupling }}$ is a term that accounts for an enhancement of the overall thermal conductivity due to a change in the local temperature gradient. The latter can be caused by the presence or variation of the other heat transfer mechanisms (e.g., local thermal shortcuts, such as the bridging of gaps between neighbouring fibres in a felt by gaseous conductivity). At a given temperature, the contributions due to transport along the solid phase or via radiation are constant and thus can be easily separated from the gas pressure dependent gaseous thermal conductivity.

The gas pressure dependence of the gaseous thermal conductivity $\lambda_{\text {gas }}$ is given by the Knudsen-formula [6] (note that the Knudsen formula was derived for the gas transport in a gap between parallel walls):

$$
\begin{aligned}
\lambda_{\mathrm{gas}} & =\frac{\lambda_{\mathrm{gas}, 0} \Phi}{(1+2 \beta K n)}=\frac{\lambda_{\mathrm{gas}, 0} \Phi}{\left(1+2 \beta \ell_{\mathrm{gas}}\left(p_{0}\right) p_{0} / p D\right)} \\
& =\frac{\lambda_{\mathrm{gas}, 0} \Phi}{\left(1+p_{1 / 2} / p\right)},
\end{aligned}
$$

with $\lambda_{\text {gas }, 0}$ the conductivity of the free gas (without convection terms!), $\beta$ a constant with a value of about 1.5 that depends on the gas and the accommodation coefficient, and $\Phi$ is the porosity of the sample. $K n$ is the Knudsen number defined as the ratio of the mean free path $l$ of the atoms or molecules in the gas at a given pressure and the effective pore diameter $D$; $p_{0}$ is a reference gas pressure (e.g. $1 \mathrm{bar}=100 \mathrm{kPa}$ ) for which $l$ is known, and $p_{1 / 2}$ is the pressure at which the value for the gaseous thermal conductivity is half of the maximum value for the free gas $\left(\Phi \lambda_{\mathrm{gas}, 0}\right)$.

Fig. 1 shows the typical gas pressure dependence of the gaseous thermal conductivity according to Eq. (2), both in the linear representation and as a lin-log plot. In the latter, the curve has a characteristic S-shape (Fig. 1b).

\subsection{Bimodal pore size distribution}

In case of a porous material with two different pore sizes $D_{1}, D_{2}$ and two contributions to the total porosity $\Phi_{1}, \Phi_{2}$, that act with respect to the heat transfer as parallel paths, the total gaseous conductivity can be written as a superposition of two terms (see Fig. 2):

$$
\begin{aligned}
\lambda_{\text {gas }, \text { total }}= & \frac{\lambda_{\text {gas }, 0} \Phi_{1}}{\left(1+2 \beta \ell_{\text {gas }}\left(p_{0}\right) p_{0} / p D_{1}\right)} \\
& +\frac{\lambda_{\text {gas }, 0,2}}{\left(1+2 \beta \ell_{\text {gas }}\left(p_{0}\right) p_{0} / p D_{2}\right)} .
\end{aligned}
$$

A pore size distribution (e.g., Gaussian type)

$$
\lambda_{\text {gas }}(D, \sigma)=\frac{1}{N} \int \exp \left(-\frac{1}{2} \frac{\left(D^{\prime}-D\right)^{2}}{\sigma^{2}}\right) \frac{\lambda_{\text {gas }, 0}}{\left(1+2 \beta l(p) / D^{\prime}\right)} \mathrm{d} D^{\prime}
$$



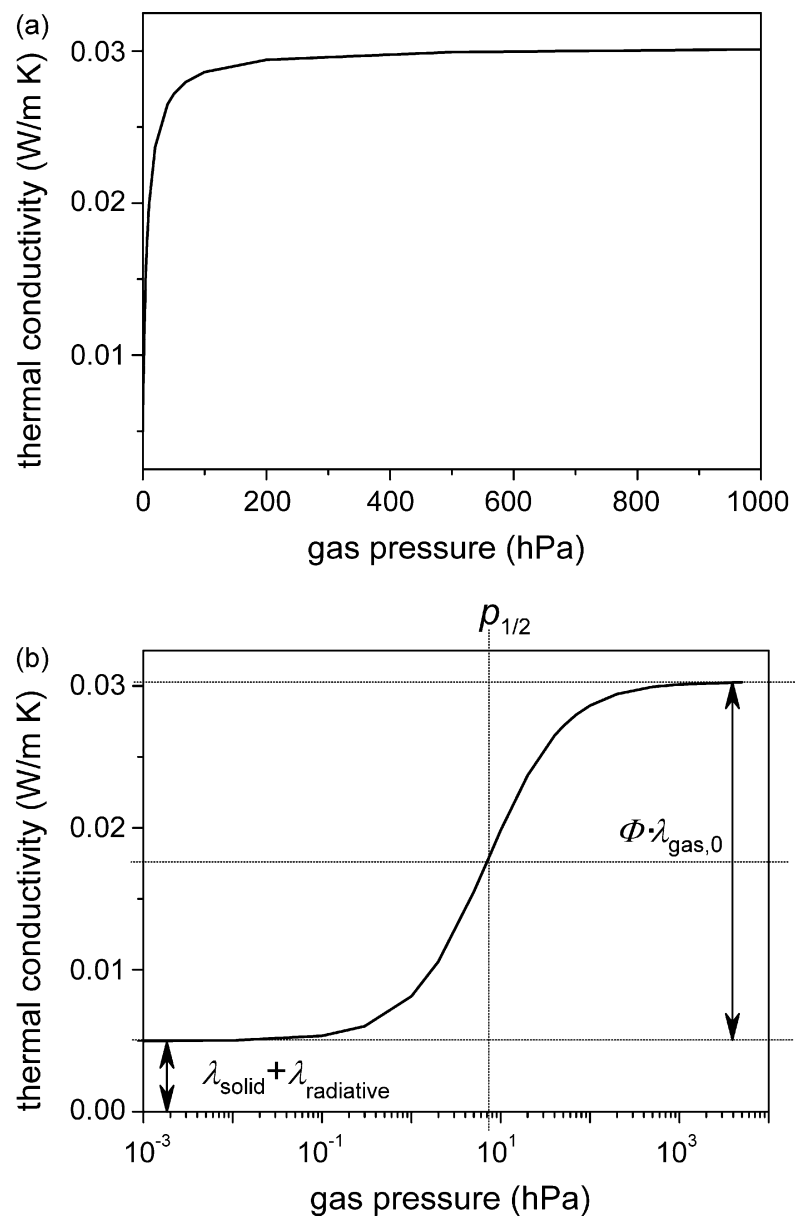

Fig. 1. Gas pressure dependence at RT of the thermal conductivity (Knudsen formula) in a linear (Fig.1b)) and half-logarithmic (Fig. 1b)) representation (pore size: about $3 \mu \mathrm{m}$ ). In the half-log plot (bottom) the different contributions can easily be separated: the offset corresponds to the thermal transport along the solid backbone of the sample and via radiative heat transfer; the gaseous contribution shows a S-shaped curve characterized by the pressure $p_{1 / 2}$ at $\left(\Phi \lambda_{\mathrm{gas}, 0}\right) / 2$.

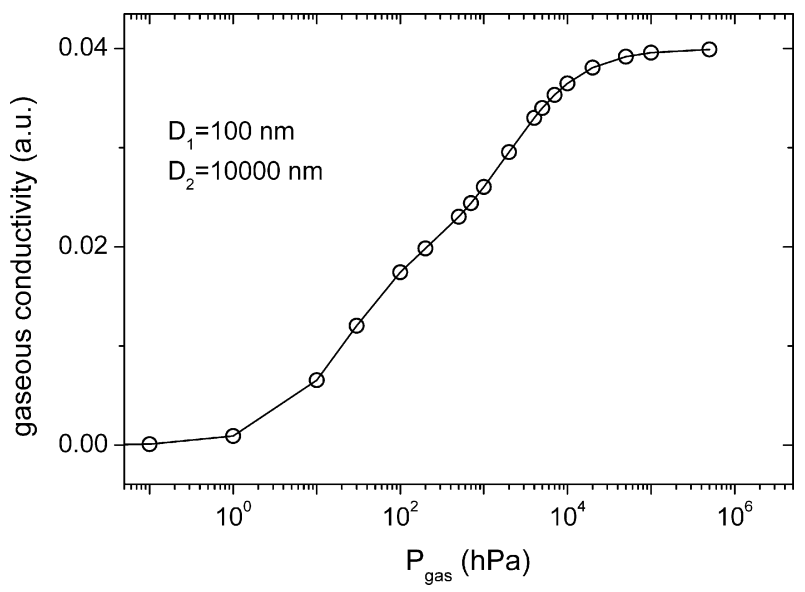

Fig. 2. Theoretical curve for a system consisting of two different pore sizes $\left(D_{1}=100 \mathrm{~nm}, D_{2}=10,000 \mathrm{~nm}\right)$, however, the same pore volume $\left(\phi_{1}=\phi_{2}\right)$. The curve represents the superposition of two independent contributions (parallel heat transport).
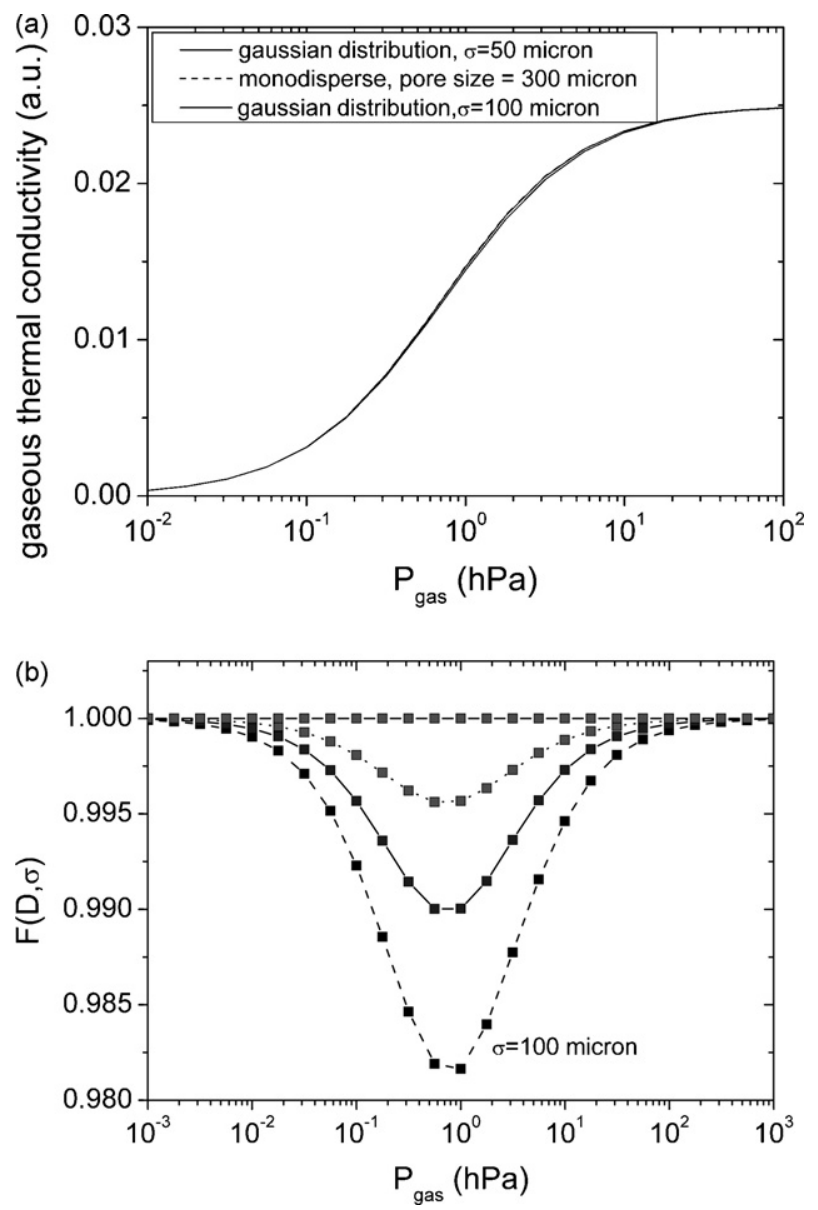

Fig. 3. (a): theoretical curve for systems with a single pore size of $300 \mu \mathrm{m}$ and a Gaussian pore volume distributions (Eq. (4)), respectively. (b): ratio $F(D, \sigma)$ of the thermal conductivities calculated for a Gaussian pore volume distribution and a monodisperse system. The minimum of the function $F$ becomes more pronounced as $\sigma$ increases from 0 to $1 / 3$ of the average pore size $D$.

results in a broadening of the gas pressure dependence; here, $D^{\prime}$ is the convolution variable, $\sigma$ the width of the Gaussian and $N$ a factor that normalizes the integral to provide the correct total porosity. In case of an average pore size of $300 \mu \mathrm{m}$ and a width $\sigma$ of the Gaussian between 50 and $100 \mu \mathrm{m}$ this effect is barely visible in the linear-log representation (Fig. 3a). If a new function $F$ is defined by normalizing the curves to the function for the monodisperse case;

$F(D, \sigma)=\lambda_{\text {gas }}(D, \sigma) \frac{(1+2 \beta l(p) / D)}{\lambda_{\text {gas }, 0}}$

the increasing broadening of the pore size distribution is reflected in an increasing value for the minimum of the function $F(D, \sigma)$ (Fig. 3b).

\section{Experimental techniques}

\subsection{Thermal conductivity}

For the measurement of the thermal conductivity different methods like hot-wire, laser-flash and hot-plate techniques are 
available (Fig. 4). Depending on the amount of sample available, the sample geometry, isotropy and characteristic size of the structural subunits (e.g. pores in a porous materials) some of these methods are more suitable and/or accurate than the other ones. Therefore the thermal conductivity was determined via a laser-flash measurement for the monolithic porous samples and by a guarded hot-plate measurement for the foam, the powders and the granules. In contrast to the guarded hot-plate setup applied, the laser-flash instrument does not allow for the application of an external load. While laser-flash and hot-wire are dynamic techniques, and therefore relatively fast, the hot-plate measurement is a time consuming, but very accurate, steady state method that can also be applied to investigate larger systems and thus samples with a substructures in the mm-range and above.

In contrast, only a small disk shaped sample with a diameter of about $10 \mathrm{~mm}$ and thickness of about $1 \mathrm{~mm}$ is necessary for a laser-flash measurements. Hot-wire equipments require sample volumes on the order of $100 \mathrm{~cm}^{3}$.

\subsubsection{Hot-wire method}

For the hot-wire measurement, a wire is integrated into a sample. This can be achieved either by preparing a sandwiched sample/wire/sample system (see Fig. 4) or by filling a chamber that already contains the clamped wire with the powdered or granular material under investigation.

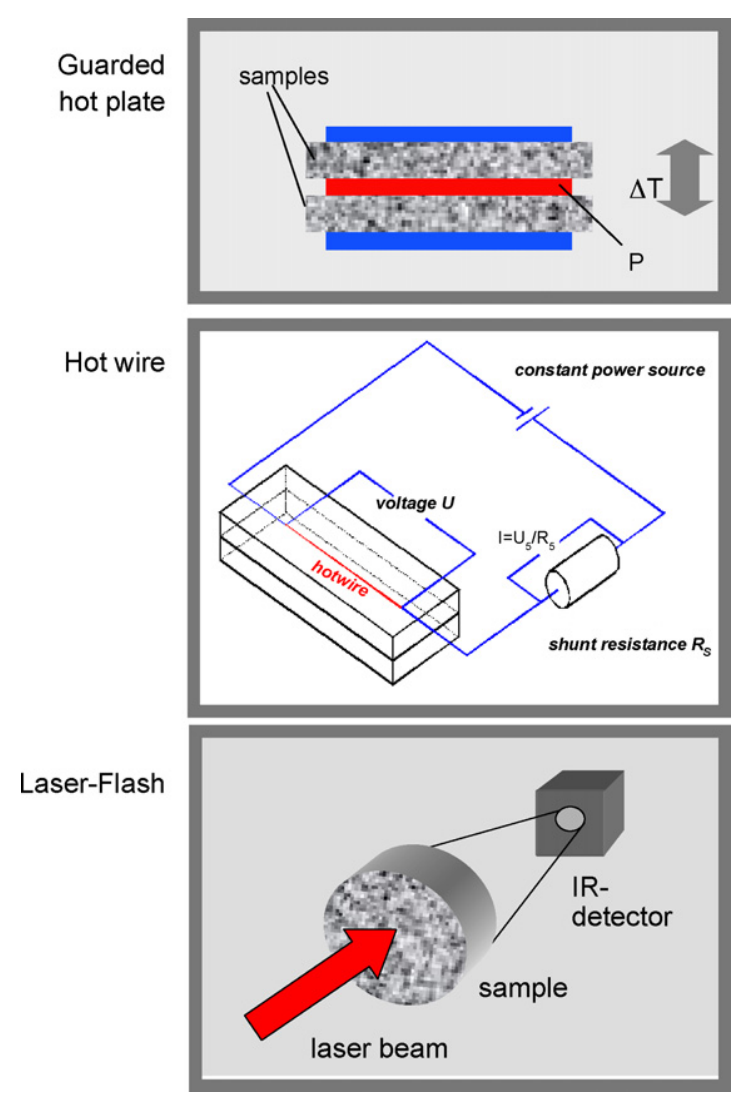

Fig. 4. Schemes of the three methods applied for the measurement of the thermal conductivity and diffusivity, respectively.
During the experiment, the wire is heated with a constant electrical power source. The wire simultaneously serves as a temperature sensor. The temperature is calculated from the increase of the temperature-dependent electrical resistance of the wire with time. The thermal conductivity is determined by evaluating the temperature rise in the wire, which depends on the thermal conductivity of the sample surrounding it. For details see, e.g. Ref. [7].

\subsubsection{Guarded hot-plate apparatus}

The main elements of the guarded hot-plate setup [8] are a central plate that can be heated, two layers of the sample with a well defined thickness located symmetrically on either side of the central plate, and two plates that work as heat sinks (Fig. 4). In case of the apparatus LOLA4 [8] the central plate consists of a circular metal plate. The heating power for the central plate is regulated such that the temperature difference (that is small compared to the average temperature of the sample) across the samples is constant. The electrical energy heats the central plate, and heat flows from there symmetrically through the two samples. To ensure a one-dimensional heat flow, the central hot plate is enclosed by two concentric guard rings that are kept at the same temperature as the central plate.

At steady state, the thermal conductivity $\lambda$ of the sample is determined from the electrical power $P^{\prime}$ that has to be provided to ensure a given temperature gradient $\Delta T$ across the samples, the mean sample thickness $d_{0}$, and the sample area in touch with the central plate $A$ :

$\lambda=\frac{P^{\prime} d_{0}}{2 A \Delta T}$.

\subsubsection{Laser-flash}

With the laser-flash method (see, e.g. Ref. [9]) one side of the sample is heated via a short laser pulse (Fig. 4). The heat diffuses through the sample and results in a rise of the temperature on the rear side of the sample that is monitored with an IR-detector. The change of the rear temperature with time is fitted with the solution of the differential equation for the given boundary conditions; the free parameter is the thermal diffusivity $a$. This quantity is related to the thermal conductivity $\lambda$ by:

$a=\frac{\lambda}{\rho c_{\mathrm{p}}}$

To calculate the thermal conductivity, the density $\rho$ and the specific heat $c_{\mathrm{p}}$ of the sample have to be known or determined separately. Within the framework of this paper the density was determined from the weight of a degassed sample and its dimensions; the specific heat was measured with a differential scanning calorimeter.

To study the gas pressure dependence of the thermal conductivity with any of the three techniques, the setups were enclosed in an evacuable chamber and thoroughly evacuated. During analysis, the pressure of an inert gas (nitrogen) is stepwise increased up to 1 bar. 
The experimental uncertainty of the three experimental methods is $3-8 \%$, with the biggest contributions coming from the sample thickness in the case of the guarded hot-plate and laser-flash measurements, the uncertainty in the determination of the density and/or an inhomogeneity of the sample. The laser-flash data furthermore include the uncertainty from the measurement of the specific heat. For a given sample, the values measured at different gas pressures should, however, possess a far higher accuracy relatively to each other, since all other factors (density, sample thickness and so on) are unchanged.

\subsection{Morphological properties of the samples}

The samples were characterized by their density, determined from their macroscopic volume and weight, and their mean pore size. The latter quantity was derived in one case via imaging analysis. For most of the samples the relation

$d=\frac{4 V_{\mathrm{P}}}{S_{\mathrm{ext}}}=\frac{4}{S_{\mathrm{ext}}}\left(\frac{1}{\rho}-\frac{1}{\rho_{\text {skeleton }}}\right)$

was applied, with the external specific surface area $S_{\text {ext }}$ determined from nitrogen sorption (ASAP 2000 with 2010 software, Micromeritics) via a t-plot or small angle X-ray scattering (SAXS) data (see, e.g. Ref. [10,11]); the scattering measurements were performed with synchrotron radiation at the instrument JUSIFA (Hasylab, Hamburg, Germany). $V_{\mathrm{p}}$ is the meso- and/or macro-pore volume, respectively, and $\rho$ and $\rho_{\text {skeleton }}$ are the bulk density of the sample and the density of the solid backbone of the material under investigation.

\section{Samples investigated}

Within the framework of this paper, samples investigated with respect to their thermal conductivity in the past for different applications were compiled. The intention was to compare the mean pore size determined from the gas pressure dependence of the thermal conductivities via Eq. (2) for materials with different types of morphology and chemical composition to the pore sizes derived from SAXS and nitrogen sorption, respectively.

The samples investigated are (see also Fig. 5):

- a system of solid glass spheres with a diameter of $1 \mathrm{~mm}$,

- an open-porous PU-foam with a pore size of about $60 \mu \mathrm{m}$,

- a sample consisting of silica aerogel granules with a broad and a narrow (1-2 $\mathrm{mm}$ ) granule diameter distribution, respectively, and a mean pore size within the granules of about $30 \mathrm{~nm}$,

- fumed silica powder (HDK by Wacker, Germany with a specific surface area of about $300 \mathrm{~m}^{2} / \mathrm{g}$ ) mixed with a few volume percent fibers and opacifying additive,

- a monolithic silica aerogel with a mean pore size of about $70 \mathrm{~nm}$,

- two carbon aerogels with a mean pore size of about 1 micrometer and $100 \mathrm{~nm}$, respectively.

\section{Experimental data}

Figs. 6-8 show the thermal conductivities as a function of gas pressure for the samples investigated. The lines through the experimental data points represent in each case a fit via Eq. (2) with the average pore size $D$ and $\lambda_{\text {gas }, 0}$ as free parameters. For the samples with small pores, where only part of the gas pressure dependence of the thermal conductivity can be measured at pressures below 1 bar, $\lambda_{\text {gas }, 0}$ was set to be $\lambda_{\text {free } \mathrm{N}_{2}}\left(\lambda_{\text {free } \mathrm{N}_{2}}=0.026 \mathrm{~W} /(\mathrm{m} \mathrm{K})\right.$ at RT). In case of the granular materials, the data were fitted with a superposition of two independent gas-pressure-dependent contributions (Eq. (3)). The average pore diameters derived via the fit and the data deduced from nitrogen sorption and SAXS are compiled in Table 1. In Fig. 9 these data, i.e. the pore sizes determined via other methods, are plotted versus the average pore size deduced from the gas pressure dependence of the thermal conductivity.

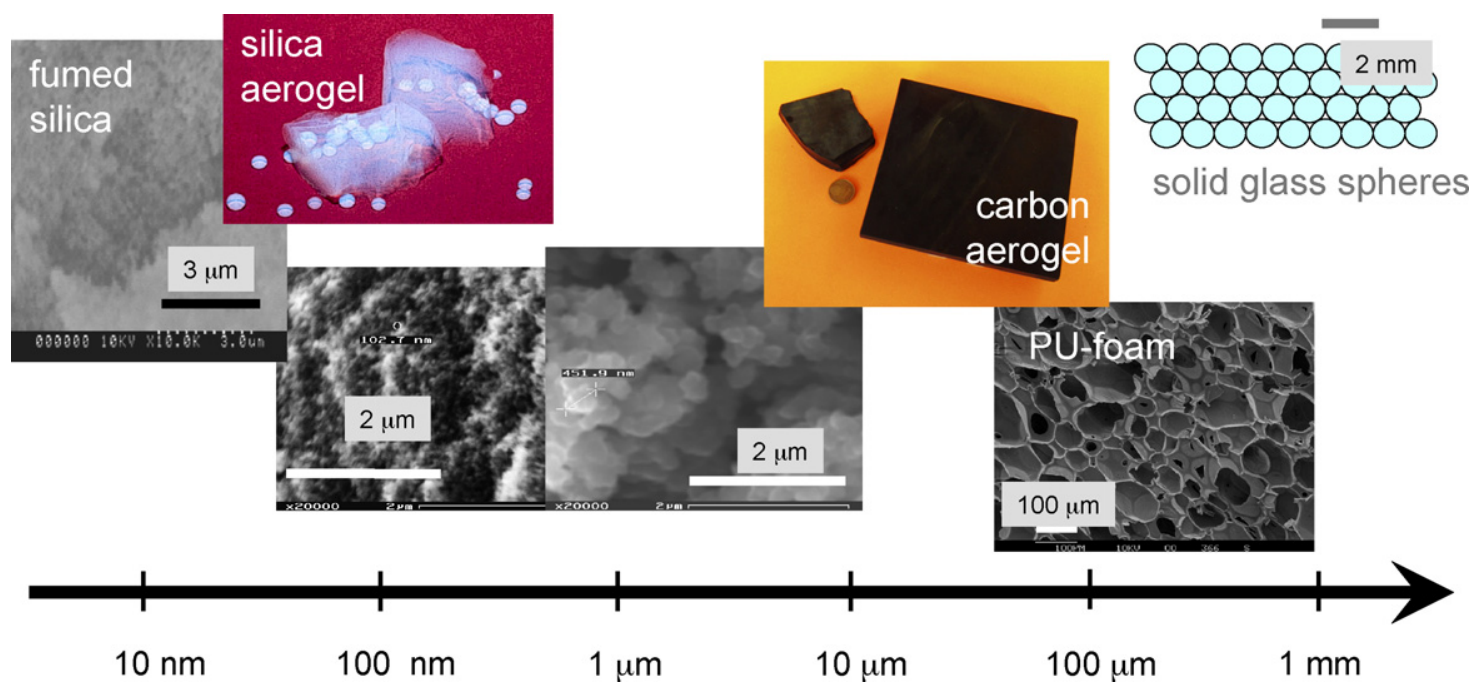

Fig. 5. Samples investigated ranging from about $100 \mathrm{~nm}$ to $1 \mathrm{~mm}$ in pore size. 
Table 1

Density, porosity and average pore size derived via the analysis of the gas pressure dependence of the thermal conductivity $(D$, Eq. (2)), sorption data $(d)$ and SAXS $\left(d_{\mathrm{SAXS}}\right)$ for different types of samples

\begin{tabular}{|c|c|c|c|c|}
\hline Sample & Density $\left(\mathrm{g} / \mathrm{cm}^{3}\right)$, porosity $(\%)$ & $D(\mathrm{~nm})$ & $d=4 V_{\mathrm{p}} / S_{\mathrm{ext}}(\mathrm{nm})$ & $d_{\text {SAXS }}(\mathrm{nm})$ \\
\hline Fumed silica & $0.162,93$ & $300 \pm 50$ & 100 & 115 \\
\hline $\mathrm{SiO}_{2}$-aerogel & $0.087,96$ & $100 \pm 10$ & 73 & 65 \\
\hline C-aerogel \#1 & $0.344,75$ & $85 \pm 30$ & 108 & $73 \mathrm{~nm}$ \\
\hline \multicolumn{5}{|l|}{$\mathrm{SiO}_{2}$ aerogel granules } \\
\hline Narrow granule SD & $0.130,94$ (0.2 granule density) & $(2.3 \pm 0.4) \times 10^{6}<150$ & $4 \times 10^{6 \mathrm{~b}} 35 \pm 5 \mathrm{~nm}$ & $\mathrm{Nd}$ \\
\hline Hard glass spheres (diameter: $1 \mathrm{~mm}$ ) & $\mathrm{Nd}$ & $(4.95 \pm 0.62) \times 10^{6}$ & $\mathrm{Nd}$ & $\mathrm{Nd}$ \\
\hline
\end{tabular}

${ }^{a}$ Meso- and macro-porosity.

b Calculated from the average spherules size (sieved).

${ }^{c}$ Determined from SEM via imaging.

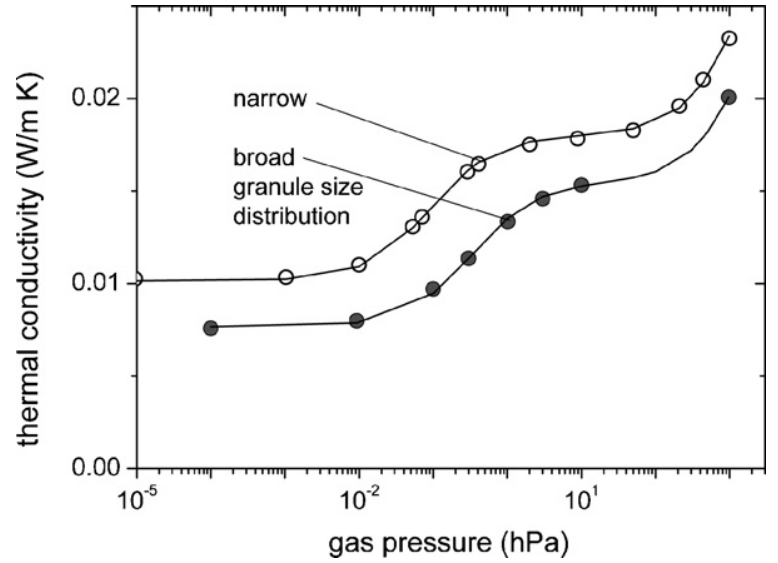

Fig. 6. Thermal conductivity as a function of nitrogen pressure at RT for two granular silica aerogels with a broad and a narrow granule size distribution. The full lines represent the fits of the data with Eq. (3).

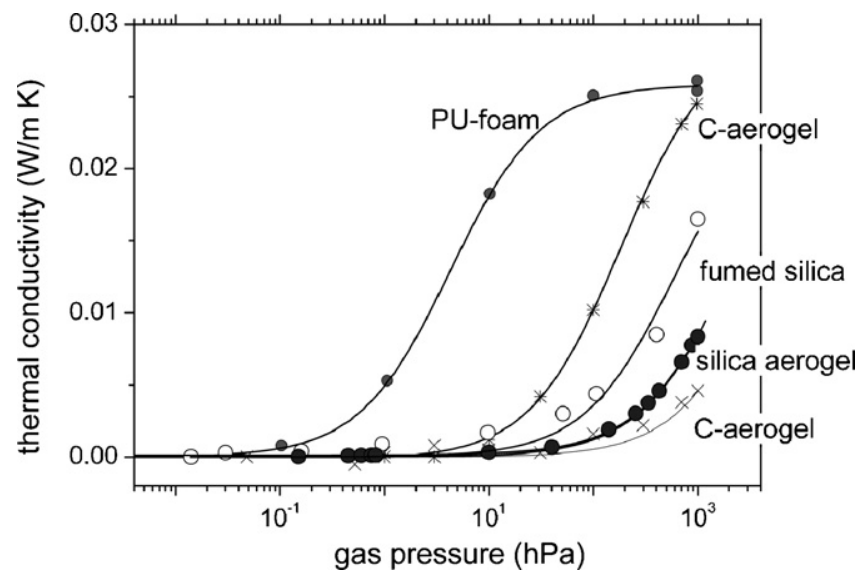

Fig. 7. Thermal conductivity as a function of nitrogen pressure at RT for different open porous materials. The full lines represent the fits of Eq. (2) to the data.

\section{Discussion}

All data, except for the hard spheres, show good agreement between the fit and the experimental thermal conductivity data as a function of gas pressure.

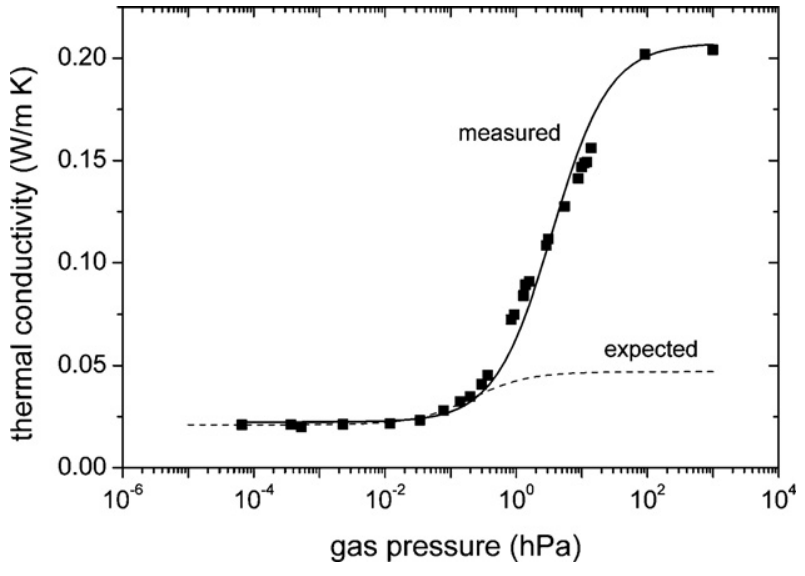

Fig. 8. Thermal conductivity as a function of nitrogen pressure at RT for solid glass spheres (diameter $1 \mathrm{~mm}$ ). The full line represents a fit of the data with Eq. (2). The dashed line is the contribution expected for a simple superposition of heat transfer via the solid and the gas phase.

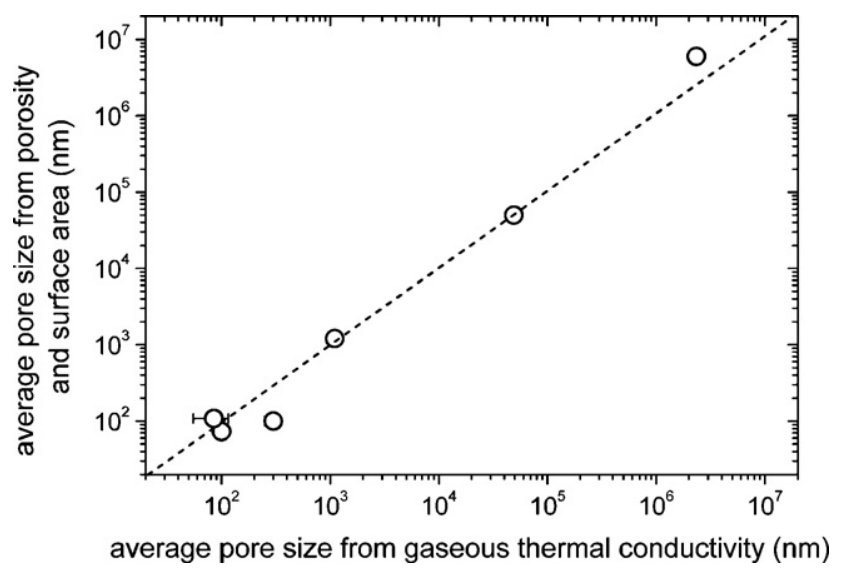

Fig. 9. Average pore size determined via sorption and imaging vs. pore size derived from the gas pressure dependence of the thermal conductivity (see also Table 1). The dashed line is a guide to the eye representing the linear relationship between the two quantities.

The thermal conductivity as a function of gas pressure of the two granular silica aerogels both show two well-separated steps that indicate porosity corresponding to a pore size on the order of 200-600 $\mu \mathrm{m}$ between the granules, and a second contribution 
due to pores on the order of $100 \mathrm{~nm}$ or below. In both cases, the height of the step at low gas pressures is about $0.0077 \mathrm{~W} /(\mathrm{m} \mathrm{K})$, a value that is equal to $30 \%$ of the value of free nitrogen. This value is close to the porosity of $26 \%$ that one can expect in a bed of packed monodisperse solid spheres. While the height of the step is very similar, the S-shaped curve for the material with the narrow granule size distribution is clearly shifted towards smaller gas pressures, indicating larger pores (see also Table 1). This trend is reasonable, since a narrow granule distribution results in larger pores between the spheres, while for a broad distribution a partial filling of gaps between larger spherules with small spheres is expected. The offsets of the curves in the low pressure limit show different contributions of thermal conductivity via the solid (silica) phase that are due to a slightly different density of the granules.

Average pore sizes derived from the gaseous thermal conductivity show a good correlation with values obtained from SAXS or sorption isotherms for materials with largely different average pore sizes, ranging from $\mathrm{mm}$ to about $100 \mathrm{~nm}$ (Fig. 9 and Table 1). This observation is confirmed by results published recently by Lee et al. [12], who investigated a series of supercritically dried polyisocyanate aerogels. They analyzed the gas pressure dependence of the thermal conductivity, measured nitrogen sorption, and examined the structure by SEM. The combination of the different methods suggests the presence of a bimodal PSD in their porous materials.

In Fig. 9 only for the fumed silica mixed with fibres and IR-opacifiers a major deviation from the dashed line can be detected: in this case, the large pore size from the thermal data results from a fit with the height of the step set to the value of the free gas $(0.025 \mathrm{~W} /(\mathrm{m} \mathrm{K}))$. Fumed silica powder exists of agglomerated solid silica particles; the porosity is provided by the free space in between the silica particles and the agglomerated silica clusters. The resulting broad pore size distribution shows in the scattering of the experimental data around the fit curve (Fig. 7); however, the experimental gas pressure range is not wide enough to separate the different contributions.

An extremely pronounced coupling effect becomes obvious in case of the solid glass spheres (Fig. 8). In this system the difference between the thermal conductivity in the evacuated state and at ambient pressure is about a factor of seven higher than expected from a superposition of gas transport and heat transfer along the touching solid glass spheres. This enhancement is due to local thermal shortcuts around the points where spheres are touching.

So far the limited number of data points does not allow the analysis of the experimental data in terms of a pore size distribution (PSD). However, Fig. 3 shows that PSD with a width of about half of the mean pore size can be identified if the experimental error is on the order of a few percent or less.

One has to keep in mind that, so far, only isotropic systems were presented and discussed. Anisotropic systems will show different thermal conductivities in the different directions and thus provide additional information about structural characteristics compared to steady state methods like sorption or porosimetry.

\section{Conclusions}

The analysis of the gaseous thermal conductivity represents a non-destructive alternative for the determination of pore sizes of materials that are deformed upon an analysis with mercury porosimetry or nitrogen sorption. Principal limitations for an application of the new approach are on the upper end of the length scale the limited typical sample dimensions in the different experimental setups; at the lower end, i.e. at small pore sizes, the experimentally accessible pressure range is the key factor. Up to now the experimental setups are mostly restricted to gas pressures below 1 bar, limiting the analysis of the gas pressure to materials with pores larger than $100 \mathrm{~nm}$. Measurements at higher pressures, however, could extend the range in accessible pore size.

To characterize materials that are applied as thermal insulation, the thermal conductivity usually is only measured at a few different gas pressures. If the data density is increased and the accuracy within a run is optimized the extraction of pore size distributions are possible.

Care has to be taken in case of systems with point contacts between stiff elements, such as fibre boards or hard spheres that will cause large artefacts.

\section{Acknowledgement}

The authors would like to thank Dr. G. Goerigk for his continuous support at the SAXS beamline JUSIFA, Hasylab.

\section{References}

[1] F.J. Broecker, W. Heckmann, F. Fischer, M. Mielke, J. Schroeder, A. Stange, Structural analysis of granular silica aerogels, in: J. Fricke (Ed.), Aerogels, Springer Proc. In Physics 6, Spinger, Berlin, Heidelberg, 1986, pp. 160-166.

[2] R. Pirard, J.P. Pirard, Aerogel compression theoretical analysis, J. NonCryst. Solids 212 (1997) 262-267.

[3] G. Reichenauer, G.W. Scherer, Nitrogen sorption in aerogels, J. Non-Cryst. Solids 285 (2001) 167-174.

[4] J. Fricke, Materials research for the optimization of thermal insulations, High Temp. High Press. 25 (1993) 379-390.

[5] R. Horn, R. Neusinger, M. Meister, J. Hetfleisch, R. Caps, J. Fricke, Switchable thermal insulations-results of computer simulations for optimisation in building applications, High Temp. High Press. 32 (2000) 669-676.

[6] M.G. Kaganer, Thermal Insulations in Cryogenic Engineering, Israel Program for Scientific Translations, 1969.

[7] H.-P. Ebert, V. Bock, O. Nilsson, J. Fricke, The hot-wire method applied to porous materials of low thermal conductivity, High Temp. High Press. 25 (1993) 391-402.

[8] U. Heinemann, J. Hetfleisch, R. Caps, J. Fricke, Evacuable Guarded Hot Plate for Thermal Conductivity Measurements between -200 and $800^{\circ} \mathrm{C}$, Eurotherm Seminar No.44, Lisbon (1995).

[9] M. Sheindlin, D. Halton, M. Musella, C. Ronchi, Advances in the use of laser-flash techniques for thermal diffusivity measurements, Rev. Sci. Instrum. 69 (1998) 1426-1436.

[10] G. Reichenauer, Micropore adsorption dynamics in synthetic carbons, Adsorption 11 (2005) 467-471.

[11] G. Reichenauer, J. Fricke, J. Manara, J. Henkel, Switching silica aerogels from transparent to opaque, J. Non-Cryst. Solids 350 (2004) 364-371.

[12] O.-J. Lee, K.-H. Lee, T.J. Yim, S.Y. Kim, K.-P. Yoo, Determination of mesopore size of aerogels from thermal conductivity measurements, J. Non-Cryst. Solids 298 (2002) 287-292. 